\title{
Probing local non-Gaussianities within a Bayesian framework
}

\author{
Franz Elsner ${ }^{1}$, Benjamin D. Wandelt ${ }^{2,3}$, and Michael D. Schneider ${ }^{4}$ \\ 1 Max-Planck-Institut für Astrophysik, Karl-Schwarzschild-Straße 1, D-85748 Garching, Germany \\ 2 Department of Physics, University of Illinois at Urbana-Champaign, 1110 W. Green Street, Urbana, IL 61801, USA \\ 3 Department of Astronomy, University of Illinois at Urbana-Champaign, 1002 W. Green Street, Urbana, IL 61801, \\ USA \\ 4 Institute for Computational Cosmology, Department of Physics, Durham University, South Road, Durham, DH1 3LE, \\ $\mathrm{UK}$
}

Received .../ Accepted ...

\begin{abstract}
Aims. We outline the Bayesian approach to inferring $f_{\mathrm{NL}}$, the level of non-Gaussianities of local type. Phrasing $f_{\mathrm{NL}}$ inference in a Bayesian framework takes advantage of existing techniques to account for instrumental effects and foreground contamination in CMB data and takes into account uncertainties in the cosmological parameters in an unambiguous way.

Methods. We derive closed form expressions for the joint posterior of $f_{\mathrm{NL}}$ and the reconstructed underlying curvature perturbation, $\Phi$, and deduce the conditional probability densities for $f_{\mathrm{NL}}$ and $\Phi$. Completing the inference problem amounts to finding the marginal density for $f_{\mathrm{NL}}$. For realistic data sets the necessary integrations are intractable. We propose an exact Hamiltonian sampling algorithm to generate correlated samples from the $f_{\mathrm{NL}}$ posterior. For sufficiently high signal-to-noise ratios, we can exploit the assumption of weak non-Gaussianity to find a direct Monte Carlo technique to generate independent samples from the posterior distribution for $f_{\mathrm{NL}}$. We illustrate our approach using a simplified toy model of CMB data for the simple case of a 1-D sky.

Results. When applied to our toy problem, we find that, in the limit of high signal-to-noise, the sampling efficiency of the approximate algorithm outperforms that of Hamiltonian sampling by two orders of magnitude. When $f_{\mathrm{NL}}$ is not significantly constrained by the data, the more efficient, approximate algorithm biases the posterior density towards $f_{\mathrm{NL}}=0$.
\end{abstract}

Key words. cosmic microwave background - cosmological parameters - Methods: data analysis - Methods: numerical Methods: statistical

\section{Introduction}

The analysis of cosmic microwave background (CMB) radiation data has considerably improved our understanding of cosmology and played a crucial role in constraining the set of fundamental cosmological parameters of the universe (Spergel et al. 2007; Hinshaw et al. 2009). This success is based on the intimate link between the temperature fluctuations we observe today and the physical processes taking place in the very early universe. Inflation is currently the favored theory predicting the shape of primordial perturbations (Guth 1981; Linde 1982), which in its canonical form leads to very small non-Gaussianities that are far from being detectable by means of present-day experiments (Maldacena 2003; Acquaviva et al. 2003). However, inflation scenarios producing larger amounts of non-Gaussianity can naturally be constructed by breaking one or more of the following properties of canonical inflation: slow-roll, single-field, Bunch-Davies vacuum, or a canonical kinetic term (Bartolo et al. 2004). Thus, a positive detection of primordial non-Gaussianity would allow us to rule out the simplest models. Combined with improving constraints on the scalar spectral index $n_{s}$, the test for non-Gaussianity is therefore complementary to the search for gravitational waves as a means to test the physics of the early Universe.

A common strategy for estimating primordial non-Gaussianity is to examine a cubic combinations of filtered CMB sky maps (Komatsu et al. 2005). This approach takes advantage of the specific bispectrum signatures produced by primordial non-Gaussianity and yields to a computationally efficient algorithm. When combined with the variance reduction technique first described by Creminelli et al. (2006) these bispectrum-based techniques are close to optimal, where optimality is defined as saturation of the Cramer-Rao bound. Lately, a more computationally costly minimum variance estimator has been implemented and applied to the WMAP5 data (Smith et al. 2009).

Recently, a Bayesian approach has been introduced in CMB power spectrum analysis and applied successfully to WMAP data making use of Gibbs-sampling techniques (Jewell et al. 2004; Wandelt et al. 2004). Within this framework, one draws samples from the posterior probability density given the data without explicitly calculating it. The target probability distribution is finally constructed out of the samples directly, thus computationally costly evaluations of

Send offprint requests to: felsner@mpa-garching.mpg.de 
the likelihood function or its derivatives are not necessary. Another advantage of the Bayesian analysis is that the method naturally offers the possibility to include a consistent treatment of the uncertainties associated with foreground emission or instrumental effects (Eriksen et al. 2008). As it is possible to model CMB and foregrounds jointly, statistical interdependencies can be directly factored into the calculations. This is not straightforward in the frequentist approach where the data analysis is usually performed in consecutive steps. Yet another important and desirable feature is the fact that a Bayesian analysis obviates the necessity to specify fiducial parameters, whereas in the frequentist approach it is only possible to test one individual null hypothesis at a time.

In this paper, we pursue the modest goal of developing the formalism for the extension of the Bayesian approach to the analysis of non-Gaussian signals, in particular to local models, where the primordial perturbations can be modeled as a spatially local, non-linear transformation of a Gaussian random field. Utilizing this method, we are able to write down the full posterior probability density function (PDF) of the level of non-Gaussianity. We demonstrate the principal aspects of our approach using a 1-D toy sky model. Although we draw our discussion on the example of CMB data analysis, the formalism presented here is of general validity and may also be applied within a different context.

The paper is organized as follows. In Sect. 2 we give a short overview of the theoretical background used to characterize primordial perturbations. We present a new approximative approach to extract the amplitude of non-Gaussianities from a map in Sect. 3 and verify the method by means of a simple synthetic data model (Sect. 4). We compare the performance of our technique to an exact Hamiltonian Monte Carlo sampler which we developer in Sect. 6 and discuss the extensions of the model required to deal with a realistic CMB sky map (Sect. 7). Finally, we summarize our results in Sect. 8,

\section{Model of non-Gaussianity}

The expansion coefficients $a_{\ell m}$ of the observed CMB temperature anisotropies in harmonic space can be related to the primordial fluctuations via

$$
a_{\ell m}=\frac{2 b_{\ell}}{\pi} \int k^{2} d k r^{2} d r\left[\Phi_{\ell m}(r) g_{\ell}^{a d i}(k)+S_{\ell m}(r) g_{\ell}^{i s o}(k)\right] j_{\ell}(k r)+n_{\ell m}
$$

where $\Phi_{\ell m}(r)$ and $S_{\ell m}(r)$ are the primordial curvature and isocurvature perturbations at comoving distance $r, g_{\ell}^{a d i}(k)$ and $g_{\ell}^{i s o}(k)$ their corresponding transfer functions in momentum space. The spherical Bessel function of order $\ell$ is denoted by $j_{\ell}(k r), b_{\ell}$ includes beam smearing effects, and $n_{\ell m}$ describes instrumental noise. As curvature perturbations dominate over isocurvature perturbations (Bean et al. 2006; Trotta 2007), we will neglect the contribution of $S_{\ell m}$ in our subsequent analysis.

Any non-Gaussian signature imprinted in the primordial perturbations will be transferred to the $a_{\ell m}$ according to Eq. 1 and is therefore detectable, in principle. Theoretical models predicting significant levels of non-Gaussian contributions to the observed signal can be subdivided into two broad classes (Babich et al. 2004): one producing non-Gaussianity of local type, the other of equilateral type. The former kind of non-Gaussianity is achieved to very good approximation in multi-field inflation as described by the curvaton model (Moroi \& Takahashi 2001; Enqvist \& Sloth 2002; Lyth et al. 2003), or in cyclic/ekpyrotic universe models (Khoury et al. 2001; Steinhardt \& Turok 2002). The latter type of nonGaussianity is typically a result of single field models with non-minimal Lagrangian including higher order derivatives (Alishahiha et al. 2004; Senatore 2005).

Concentrating on local models, we can parametrize the non-Gaussianity of $\Phi$ by introducing an additional quadratic dependence on a purely Gaussian auxiliary field $\Phi_{\mathrm{L}}$, that is local in real space, of the form (Verde et al. 2000; Komatsu \& Spergel 2001)

$$
\Phi_{\mathrm{NL}}(r)=\Phi_{\mathrm{L}}(r)+f_{\mathrm{NL}}\left[\Phi_{\mathrm{L}}^{2}(r)-\left\langle\Phi_{\mathrm{L}}^{2}(r)\right\rangle\right]
$$

where $f_{\mathrm{NL}}$ is a dimensionless measure of the amplitude of non-Gaussianity.

\section{Bayesian inference of non-Gaussianity}

It has been shown to be feasible to reconstruct the primordial curvature potential out of temperature or temperature and polarization sky maps (Yadav \& Wandelt 2005; Elsner \& Wandelt 2009), which allows searching for primordial nonGaussianities more sensitively. Although the mapping from a 3D potential to a 2D CMB sky map is not invertible unambiguously, a unique solution can be found by requiring that the result minimizes the variance. In this conventional frequentist approach, the level of non-Gaussianity and an estimate of its error is derived from a cubic combination of filtered sky maps (Komatsu et al. 2005). We will show in the following sections how to sample $f_{\mathrm{NL}}$ from the data and unveil the full posterior PDF using a Bayesian approach.

\subsection{Joint probability distribution}

In our analysis we assume the data vector $d$ to be a superposition of the CMB signal $s$ and additive noise $n$

$$
\begin{aligned}
d & =B s+n \\
& =B M \Phi+n
\end{aligned}
$$


where information about observing strategy and the optical system are encoded in a pointing matrix $B$ and $M$ is a linear transformation matrix. In harmonic space, the signal is related to the primordial scalar perturbation as

$$
\begin{aligned}
s_{\ell m} & =\frac{2}{\pi} \int k^{2} d k r^{2} d r \Phi_{\ell m}(r) g_{\ell}^{a d i}(k) j_{\ell}(k r) \\
& \approx \sum_{i} M_{i} \Phi_{\ell m}\left(r_{i}\right) \\
& \equiv M \Phi_{\ell m} .
\end{aligned}
$$

Our aim is to construct the posterior PDF of the amplitude of non-Gaussianities given the data, $P\left(f_{\mathrm{NL}} \mid d\right)$. To do so, we subsume the remaining set of cosmological parameters to a vector $\theta$ and calculate the joint distribution as

$$
P\left(d, \Phi_{\mathrm{L}}, f_{\mathrm{NL}}, \theta\right)=P\left(d \mid \Phi_{\mathrm{L}}, f_{\mathrm{NL}}, \theta\right) P\left(\Phi_{\mathrm{L}} \mid \theta\right) P(\theta) P\left(f_{\mathrm{NL}}\right) .
$$

Now, we can use Eq. 2 to express the probability for data $d$ given $\Phi_{\mathrm{L}}, f_{\mathrm{NL}}$, and $\theta$

$$
P\left(d \mid \Phi_{\mathrm{L}}, f_{\mathrm{NL}}, \theta\right)=\frac{1}{\sqrt{|2 \pi N|}} \mathrm{e}^{-1 / 2\left[d-B M\left(\Phi_{\mathrm{L}}+f_{\mathrm{NL}}\left(\Phi_{\mathrm{L}}^{2}-\left\langle\Phi_{\mathrm{L}}^{2}\right\rangle\right)\right)\right]^{\dagger} N^{-1}\left[d-B M\left(\Phi_{\mathrm{L}}+f_{\mathrm{NL}}\left(\Phi_{\mathrm{L}}^{2}-\left\langle\Phi_{\mathrm{L}}^{2}\right\rangle\right)\right)\right]},
$$

where $N$ is the noise covariance matrix. The prior probability distribution for $\Phi_{\mathrm{L}}$ given $\theta$ can be expressed by a multivariate Gaussian by definition. Using the covariance matrix $P_{\Phi}$ of the potential, we derive

$$
P\left(\Phi_{\mathrm{L}} \mid \theta\right)=\frac{1}{\sqrt{\left|2 \pi P_{\Phi}\right|}} \mathrm{e}^{-1 / 2 \Phi_{\mathrm{L}}^{\dagger} P_{\Phi}^{-1} \Phi_{\mathrm{L}}} .
$$

For flat priors $P\left(f_{\mathrm{NL}}\right), P(\theta)$ we finally obtain

$$
\begin{aligned}
P\left(d, \Phi_{\mathrm{L}}, f_{\mathrm{NL}}, \theta\right) \propto \exp \left\{-\frac{1}{2}\left[\left(d-B M\left(\Phi_{\mathrm{L}}+f_{\mathrm{NL}}\left(\Phi_{\mathrm{L}}^{2}-\left\langle\Phi_{\mathrm{L}}^{2}\right\rangle\right)\right)\right)^{\dagger} N^{-1}\right.\right. & \\
& \left.\left.\times\left(d-B M\left(\Phi_{\mathrm{L}}+f_{\mathrm{NL}}\left(\Phi_{\mathrm{L}}^{2}-\left\langle\Phi_{\mathrm{L}}^{2}\right\rangle\right)\right)\right)+\Phi_{\mathrm{L}}^{\dagger} P_{\Phi}^{-1} \Phi_{\mathrm{L}}\right]\right\}
\end{aligned}
$$

as an exact expression for the joint distribution up to a normalization factor.

To derive the posterior density, $P\left(f_{\mathrm{NL}} \mid d\right)$, one has to marginalize the joint distribution over $\Phi_{\mathrm{L}}$ and $\theta$. As it is not possible to calculate the high dimensional $\Phi_{\mathrm{L}}$ integral directly, an effective sampling scheme must be found to evaluate the expression by means of a Monte Carlo algorithm. One possibility would be to let a Gibbs sampler explore the parameter space. Unfortunately, we were not able to find an efficient sampling recipe from the conditional densities for $f_{\mathrm{NL}}$ and $\Phi_{\mathrm{L}}$ as the variables are highly correlated. An algorithm that also generates correlated samples, but is potentially suitable for non-Gaussian densities and high degrees of correlation is the Hamiltonian Monte Carlo approach. We will return to this approach in Sect. 6.

For now we attempt to go beyond correlated samplers and see whether we can develop an approximate scheme, valid in the limit of weak non-Gaussianity, to sample $f_{\mathrm{NL}}$ independently. We start out by expanding the target posterior distribution into an integral of conditional probabilities over the non-linear potential $\Phi_{\mathrm{NL}}$,

$$
P\left(f_{\mathrm{NL}} \mid d\right)=\int d \Phi_{\mathrm{NL}} d \theta P\left(f_{\mathrm{NL}} \mid \Phi_{\mathrm{NL}}, \theta\right) P\left(\Phi_{\mathrm{NL}} \mid d, \theta\right) P(\theta \mid d) .
$$

To construct the conditional probability $P\left(\Phi_{\mathrm{NL}} \mid d, \theta\right)$ in the integrand, we need to find an equivalent equation for the joint distribution (Eq. 8) as a function of the field $\Phi_{\mathrm{NL}}$. However, a simple analytic expression for the prior distribution of $\Phi_{\mathrm{NL}}$ does not exist because it is a non-linear transform of the Gaussian auxiliary field $\Phi_{\mathrm{L}}$. To quantify the expected correction, we calculate its covariance matrix,

$$
\begin{aligned}
\left(P_{\Phi_{\mathrm{NL}}}\right)_{i j} & =\left\langle\left(\Phi_{\mathrm{NL}}\right)_{i}\left(\Phi_{\mathrm{NL}}\right)_{j}\right\rangle \\
& =\left\langle\left(\Phi_{\mathrm{L}}\right)_{i}\left(\Phi_{\mathrm{L}}\right)_{j}\right\rangle+f_{\mathrm{NL}}^{2}\left[\left\langle\left(\Phi_{\mathrm{L}}\right)_{i}^{2}\left(\Phi_{\mathrm{L}}\right)_{j}^{2}\right\rangle-\left\langle\left(\Phi_{\mathrm{L}}\right)_{i}^{2}\right\rangle\left\langle\left(\Phi_{\mathrm{L}}\right)_{j}^{2}\right\rangle\right] \\
& =\left(P_{\Phi_{\mathrm{L}}}\right)_{i j}+2 f_{\mathrm{NL}}^{2}\left(P_{\Phi_{\mathrm{L}}}\right)_{i j}^{2} .
\end{aligned}
$$

As the covariance matrix $P_{\Phi_{\mathrm{L}}}$ is of the order $\mathcal{O}\left(10^{-10}\right)$ and the non-Gaussian contribution to $\Phi_{\mathrm{NL}}$ is known to be small, we neglect the higher order correction in the prior distribution in what follows. That is, we approximate the true prior probability function by a Gaussian distribution in $\Phi_{\mathrm{NL}}$ and in this way derive a simple expression for the joint density, as a function of $\Phi_{\mathrm{NL}}$,

$$
P\left(d, \Phi_{\mathrm{NL}}, \theta\right) \propto \exp \left\{-\frac{1}{2}\left[\left(d-B M \Phi_{\mathrm{NL}}\right)^{\dagger} N^{-1}\left(d-B M \Phi_{\mathrm{NL}}\right)+\Phi_{\mathrm{NL}}^{\dagger} P_{\Phi}^{-1} \Phi_{\mathrm{NL}}\right]\right\} .
$$


Note, that the approximation applies to the second term only, the first part of the expression remains unaffected. As this approximation is equivalent to imposing the prior belief of purely Gaussian primordial perturbations, we expect to underestimate $f_{\mathrm{NL}}$ in the low signal-to-noise regime, as we tend to replace the Wiener filtered noise with purely Gaussian signal. Contrary, the method is unbiased when the likelihood dominates over the prior which is unlikely for data derived by the Planck mission.

The direct evaluation of the joint distributions over a grid in the high dimensional parameter space is computationally not feasible. One option would be to approximate the PDF around its maximum to get an expression for the attributed errors (Tegmark 1997; Bond et al. 1998). These methods are still computationally expensive and can also not recover the full posterior. An alternative approach to overcome these problems is to draw samples from the PDF which is to be evaluated as we will discuss in the next section.

\subsection{Conditional probabilities}

To construct the target posterior density Eq. 9. we have to find expressions for the conditional probabilities $P\left(\Phi_{\mathrm{NL}} \mid d, \theta\right)$ and $P\left(f_{\mathrm{NL}} \mid \Phi_{\mathrm{NL}}, \theta\right)$. The former distribution can easily be derived from the joint probability density Eq. 11 , Since the exponent is quadratic in $\Phi_{\mathrm{NL}}$ in our approximation, the conditional PDF of $\Phi_{\mathrm{NL}}$ given $d$ and $\theta$ is Gaussian. Therefore, we can calculate mean and variance of the distribution via differentiating the expression,

$$
\begin{aligned}
\left\langle\Phi_{\mathrm{NL}}\right\rangle & =\left\langle\left(\Phi_{\mathrm{NL}}-\left\langle\Phi_{\mathrm{NL}}\right\rangle\right)^{2}\right\rangle M^{\dagger} B^{\dagger} N^{-1} d \\
\left\langle\left(\Phi_{\mathrm{NL}}-\left\langle\Phi_{\mathrm{NL}}\right\rangle\right)^{2}\right\rangle & =\left[M^{\dagger} B^{\dagger} N^{-1} B M+P_{\Phi}^{-1}\right]^{-1} .
\end{aligned}
$$

As a next step, we derive the conditional probability distribution of $f_{\mathrm{NL}}$ for given $\Phi_{\mathrm{NL}}$. This expression is not affected by the approximation and can be derived from a marginalization over $\Phi_{\mathrm{L}}$,

$$
\begin{aligned}
P\left(f_{\mathrm{NL}} \mid \Phi_{\mathrm{NL}}, \theta\right) & =\int d \Phi_{\mathrm{L}} P\left(f_{\mathrm{NL}} \mid \Phi_{\mathrm{L}}, \Phi_{\mathrm{NL}}\right) P\left(\Phi_{\mathrm{L}} \mid \theta\right) \\
& =\int d \Phi_{\mathrm{L}} \delta\left(\Phi_{\mathrm{NL}}-\Phi_{\mathrm{L}}-f_{\mathrm{NL}}\left(\Phi_{\mathrm{L}}^{2}-\left\langle\Phi_{\mathrm{L}}^{2}\right\rangle\right)\right) P\left(\Phi_{\mathrm{L}} \mid \theta\right) .
\end{aligned}
$$

Using Eq. 17, we can calculate the integral and obtain

$$
P\left(f_{\mathrm{NL}} \mid \Phi_{\mathrm{NL}}, \theta\right) \propto\left|\prod_{i} \frac{1}{1+2 f_{\mathrm{NL}}\left(\tilde{\Phi}_{\mathrm{L}}\right)_{i}}\right| \mathrm{e}^{-1 / 2 \tilde{\Phi}_{\mathrm{L}}^{\dagger} P_{\Phi}^{-1} \tilde{\Phi}_{\mathrm{L}}}
$$

where $\tilde{\Phi}_{\mathrm{L}}$ is a function of $f_{\mathrm{NL}}$ and can be regarded as inversion of Eq. 2

$$
\tilde{\Phi}_{\mathrm{L}}=\frac{1}{2 f_{\mathrm{NL}}}\left[-1+\sqrt{1+4 f_{\mathrm{NL}}\left(\Phi_{\mathrm{NL}}+f_{\mathrm{NL}}\left\langle\Phi_{\mathrm{L}}^{2}\right\rangle\right)}\right] .
$$

Note that we can resolve the ambiguity in sign in the weakly non-Gaussian limit (Babich 2005). Because the absolute value of the elements of the second solution is typically larger by orders of magnitude, the probability of its realization is strongly disfavored by the prior $P\left(\Phi_{\mathrm{L}}\right)$. The factor of suppression is typically less than $10^{-1000}$ and further vanishing with decreasing $f_{\mathrm{NL}}$.

After setting up the conditional densities, we now can sample from the distributions iteratively. First, we draw $\Phi_{\mathrm{NL}}$ from a Gaussian distribution using Eqs. 12. Then, $f_{\mathrm{NL}}$ can be sampled according to Eq. 14 using the value of $\Phi_{\mathrm{NL}}$ derived in the preceding step. Thus, the sampling scheme reads as

$$
\begin{aligned}
\Phi_{\mathrm{NL}}^{i} & \hookleftarrow P\left(\Phi_{\mathrm{NL}} \mid d, \theta\right) \\
f_{\mathrm{NL}}^{i} & \hookleftarrow P\left(f_{\mathrm{NL}} \mid \Phi_{\mathrm{NL}}^{i}, \theta\right) .
\end{aligned}
$$

Note that this is not Gibbs sampling. For a fixed set of cosmological parameters, we can chain together samples from the conditional densities above, producing independent $f_{\mathrm{NL}}$ samples. The efficiency of such a direct Monte Carlo sampler is therefore expected to be much higher than that of a Gibbs sampler, which, in the general case, would produce correlated samples.

As an extension of the sampling scheme presented so far, we sketch an approach to account for uncertainties in cosmological parameters and foreground contributions. Complementing the scheme (Eqs. 16) by an additional step allows to take into account the error in the parameters $\theta$,

$$
\begin{aligned}
\Phi_{\mathrm{NL}}^{i} & \hookleftarrow P\left(\Phi_{\mathrm{NL}} \mid d, \theta^{i-1}\right) \\
f_{\mathrm{NL}}^{i} & \hookleftarrow P\left(f_{\mathrm{NL}} \mid \Phi_{\mathrm{NL}}^{i}, \theta^{i-1}\right) \\
\theta^{i} & \hookleftarrow P\left(\theta \mid d, f_{\mathrm{NL}}^{i}\right),
\end{aligned}
$$


where the last equation updates the cosmological parameters that can be sampled from the data by means of standard Monte Carlo analysis tool: 1 . Now, the scheme formally reads as a Gibbs sampler and can in principle take into account the correlation among $f_{\mathrm{NL}}$ and the other cosmological parameters exactly. In practice, however, the impact of a non-vanishing $f_{\mathrm{NL}}$ is expected to be negligible, i.e. $P\left(\theta \mid d, f_{\mathrm{NL}}\right) \approx P(\theta \mid d)$. Likewise, we can allow for an additional sampling step to deal with foreground contributions, e.g. from synchrotron, free-free, and dust emission. Foreground templates $f^{s y n c}$, free, dust, that are available for these sources, can be subtracted with amplitudes $c^{\text {sync, free, dust }}$ which are sampled from the data in each iteration, $c^{i} \hookleftarrow P\left(c \mid d, f^{s y n c}\right.$, free, dust,$\theta^{i}$ ) (Wandelt et al. 2004). Alternatively, component separation techniques could be used to take foreground contaminants into account without the need to rely on a priori defined templates (Eriksen et al. 2006). The traditional approach to deal with point sources is to mask affected regions of the sky to exclude them from the analysis. Discrete object detection has been demonstrated to be possible within a Bayesian framework (Hobson \& McLachlan 2003; Carvalho et al. 2009), and can be fully included into the sampling chain. However, as sources are only successfully detected down to an experiment-specific flux limit, a residue-free removal of their contribution is in general not possible.

As the angular resolution of sky maps produced by existing CMB experiments like WMAP is high and will further increase once data of the Planck satellite mission becomes available, computational feasibility of an analysis tool is an issue. The speed of our method in a full implementation is limited by harmonic transforms which scale as $\mathcal{O}\left(N_{\text {pix }}^{3 / 2}\right)$ and are needed to calculate the primordial perturbations at numerous shells at distances from the cosmic horizon to zero. Thus, it shows the same scaling relation as fast cubic estimators (Komatsu et al. 2005; Yadav et al. 2007), albeit with a larger prefactor.

\section{Implementation and Discussion}

To verify our results and demonstrate the applicability of the method, we implemented a simple 1-D toy model. We considered a vector $\Phi_{\mathrm{L}}$ of random numbers generated from a heptadiagonal covariance matrix with elements

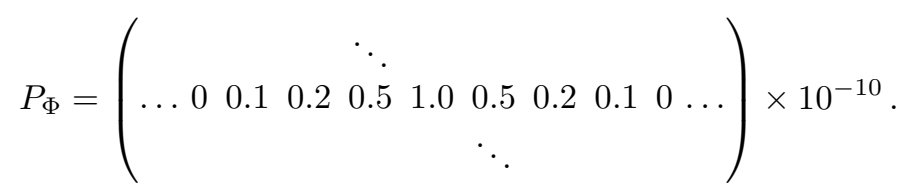

Then, a data vector with weak non-Gaussianity according to Eq. 2 was produced and superimposed with Gaussian white noise. Constructed in this way, it is of the order $\mathcal{O}\left(10^{-5}\right)$, thus the amplitude of the resulting signal $s$ is comparable to CMB anisotropies.

The data vector had a length of $10^{6}$ pixels; for simplicity, we set the beam function $B$ and the linear transformation matrix $M$ to unity. This setup allows a brute force implementation of all equations at a sufficient computational speed. We define the signal-to-noise ratio $(S / N)$ per pixel as the standard deviation of the input signal divided by the standard deviation of the additive noise. It was chosen in the range $0.5-10$ to model the typical $\mathrm{S} / \mathrm{N}$ per pixel of most CMB experiments. To reconstruct the signal, we draw 1000 samples according to the scheme in Eq. 16 .

Whereas the $\Phi_{\mathrm{NL}}$ can be generated directly from a simple Gaussian distribution with known mean and variance, the construction of the $f_{\mathrm{NL}}$ is slightly more complex. For each $\Phi_{\mathrm{NL}}$, we ran a Metropolis Hastings algorithm with symmetric Gaussian proposal density with a width comparable to that of the target density and started the chain at $f_{\mathrm{NL}}=0$. We run the $f_{\mathrm{NL}}$ chain to convergence. We ensured that after ten accepted steps the sampler has decorrelated from the starting point. Our tests conducted with several chains run in parallel give $1<R<1.01$, where $\mathrm{R}$ is the convergence statistic proposed by Gelman \& Rubin (1992). We record the last element of the chain as the new $f_{\mathrm{NL}}$ sample.

Finally, we compared the obtained sets of values $\left\{\Phi_{\mathrm{NL}}^{i}\right\},\left\{f_{\mathrm{NL}}^{i}\right\}$ to the initial data. An example is shown in Fig. [1 where we illustrate the reconstruction of a given potential $\Phi_{\mathrm{NL}}$ for different signal-to-noise ratios per pixel. The $1-\sigma$ error bounds are calculated from the $16 \%$ and $84 \%$ quantile of the generated sample. Typical posterior densities for $f_{\mathrm{NL}}$ as derived from the sample can be seen in Fig. 2. We considered the cases $f_{\mathrm{NL}}=0$ and $f_{\mathrm{NL}}=200$ with $S / N=10$ per pixel and show the distributions generated from 1000 draws. The derived posterior densities possesses a mean value of $f_{\mathrm{NL}}=6 \pm 40$ and $f_{\mathrm{NL}}=201 \pm 40$, respectively. The width of the posterior is determined by both the shape of the conditional PDF of $f_{\mathrm{NL}}$ for a given $\Phi_{\mathrm{NL}}$ and the shift of this distribution for different draws of $\Phi_{\mathrm{NL}}$ (Fig. 31). The analysis of several data sets indicate that the approximation does not bias the posterior density if the data are decisive. We illustrate this issue in the left panel of Fig. [4 where we show the distribution of the mean values $\left\langle f_{\mathrm{NL}}\right\rangle$ of the posterior density constructed from 100 independent simulations. For an input value of $f_{\mathrm{NL}}=200$ we derive a mean value $\left\langle f_{\mathrm{NL}}\right\rangle=199.3 \pm 34.8$ and conclude that our sampler is unbiased for these input parameters. For a high noise level, however, the $\Phi_{\mathrm{NL}}$ can always be sampled such that they are purely Gaussian fields and thus the resulting PDF for $f_{\mathrm{NL}}$ is then shifted towards $f_{\mathrm{NL}}=0$. This behavior is demonstrated in Fig. 5 where we compare the constructed posterior density for the cases $S / N=10$ and $S / N=0.5$ per pixel. If the noise level becomes high, the approximated prior distribution dominates and leads to both, a systematic displacement and an artificially reduced width of the posterior. Therefore, the sampler constructed here is conservative in a sense that it will tend to underpredict the value of $f_{\mathrm{NL}}$ if the data are ambiguous.

\footnotetext{
1 E.g. as described in Lewis \& Bridle (2002)
} 

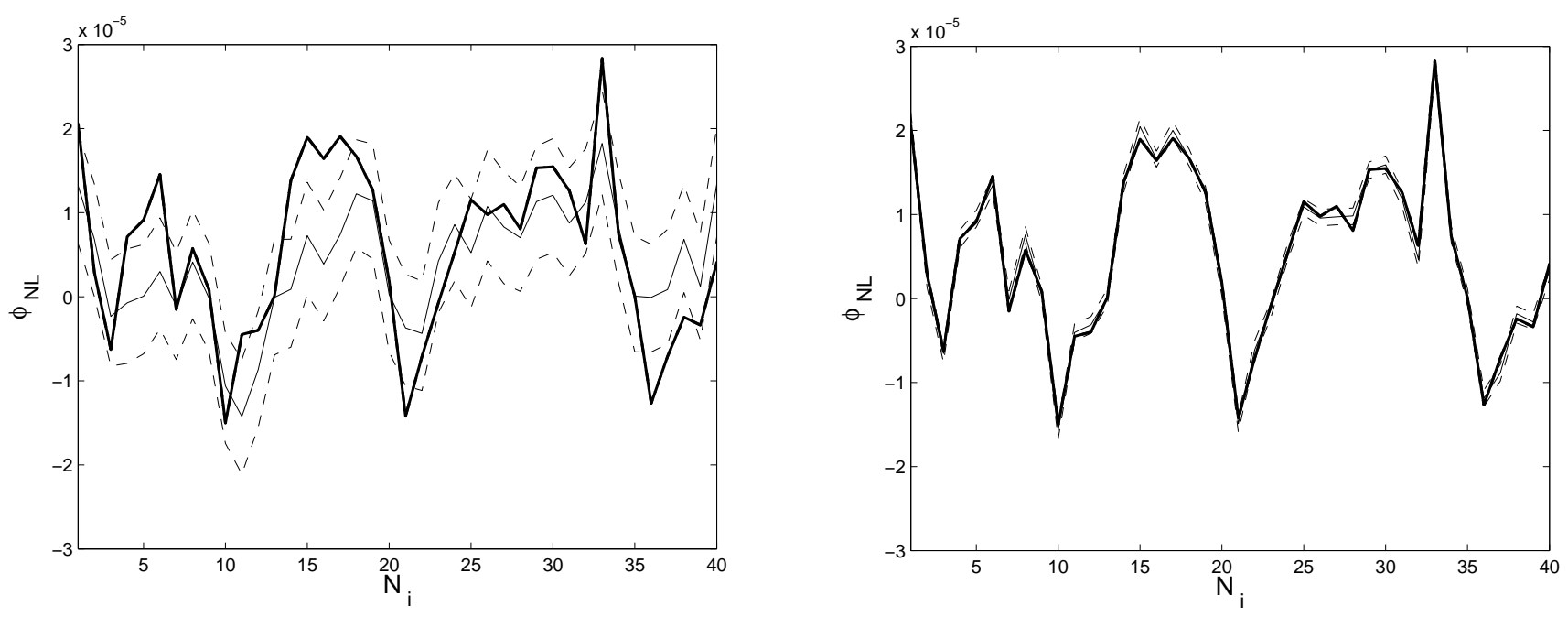

Fig. 1. Examples of reconstructed potentials $\Phi_{\mathrm{NL}}$. Left panel: The input parameters for the calculation were $f_{\mathrm{NL}}=200$ and $S / N=1$. Right panel: Analysis of the same data set for a signal-to-noise ratio of $S / N=10$. For clarity, we show only 40 elements of the $\Phi_{\mathrm{NL}}$-vector (thick solid line) and its reconstruction (thin solid line) as well as the $1-\sigma$ error bounds (dashed lines). As the difference between $\Phi_{\mathrm{NL}}$ and the linear potential $\Phi_{\mathrm{L}}$ is very small, $\Phi_{\mathrm{L}}$ can not be distinguished from $\Phi_{\mathrm{NL}}$ in this plot. In both cases 1000 samples were drawn.

An example of the evolution of the drawn $f_{\mathrm{NL}}$ samples with time can be seen in Fig. 6, where we in addition show the corresponding autocorrelation function as defined via

$$
\xi(\Delta N)=\frac{1}{N} \sum_{i}^{N} \frac{\left(f_{\mathrm{NL}}^{i}-\mu\right) \cdot\left(f_{\mathrm{NL}}^{i+\Delta N}-\mu\right)}{\sigma^{2}},
$$

where $\mathrm{N}$ is the length of the generated $f_{\mathrm{NL}}$ chain with mean $\mu$ and variance $\sigma^{2}$. The uncorrelated samples of $f_{\mathrm{NL}}$ ensure an excellent mixing of the chain resulting in a fast convergence rate.

\section{Optimality}

In a frequentist analysis, parameter inference corresponds to finding an estimator that enables to compute the most probable value of the quantity of interest as well as a bound for the error. Ideally, the estimator is unbiased and optimal, i.e. it's expectation value coincides with the true value of the parameter and the error satisfies the Cramer-Rao bound. Contrary, in a Bayesian approach, one calculates the full probability distribution of the parameter directly. Strictly speaking, optimality is therefore an ill-defined term within the Bayesian framework. All we have to show is that the approximation adopted in Eq. 11] does not affect the outcome of the calculation significantly. Note that the simplification corresponds to imposing the prior of a purely Gaussian data set. In the case of the CMB, this is a very reasonable assumption because up to now no detection of $f_{\mathrm{NL}}$ has been reported.

To investigate the effects of the approximation, we checked the dependence of the width of the posterior distribution on $f_{\mathrm{NL}}$ by running a set of simulations with varying input values $f_{\mathrm{NL}}=0,50,100,150,200,250$. The estimated standard deviation $\sigma_{f_{\mathrm{NL}}}$ of the drawn $f_{\mathrm{NL}}$ samples, each averaged over 10 simulation runs, are depicted in the right panel of Fig. 4. Contrary to the KSW estimator that shows an increase of $\sigma_{f_{\mathrm{NL}}}$ with $f_{\mathrm{NL}}$, we find no such indication of sub-optimal behavior in the relevant region of small non-Gaussianity. In particular, as the width of the distribution stays constant in the limit $f_{\mathrm{NL}} \rightarrow 0$ where our approximated equations evolve into the exact expressions, we conclude that the adopted simplification does not affect the result significantly.

This finding can also be interpreted from a different point of view: It is possible to define a frequentist estimator for $f_{\mathrm{NL}}$ based on the mean of the posterior distribution. Our results indicate that such an estimator is unbiased in the high signal-to-noise regime.

We apply an additional test in the next section where we compare our sampling algorithm to a slower but exact scheme.

\section{Hamiltonian Monte Carlo sampling}

In addition to the sampling technique presented above, we tested whether an exact Hamiltonian Monte Carlo (HMC) sampler is applicable to the problem. Within this approach one uses the methods developed in classical mechanics to describe the motion of particles in potentials. The quantity of interest is regarded as the spatial coordinate of a particle and the potential well corresponds to the PDF to evaluate (Duane et al. 1987). To each variable $\left(f_{\mathrm{NL}}, \Phi_{\mathrm{L}, 1}, \ldots, \Phi_{\mathrm{L}, n}\right)$, 

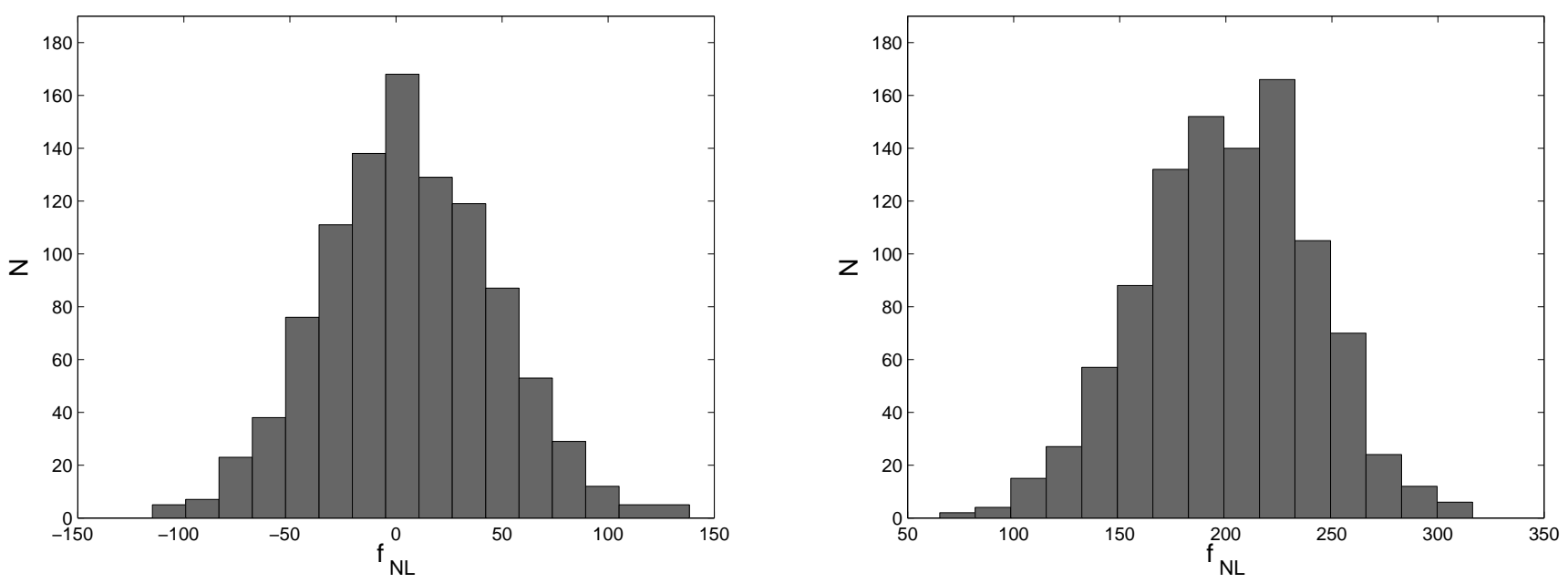

Fig. 2. Examples of a constructed posterior distribution for $f_{\mathrm{NL}}$. The input parameters used in this runs were $N_{p i x}=10^{6}$, $S / N=10$ and $f_{\mathrm{NL}}=0$ (left panel) or $f_{\mathrm{NL}}=200$ (right panel). For each parameter combination 1000 samples were drawn.
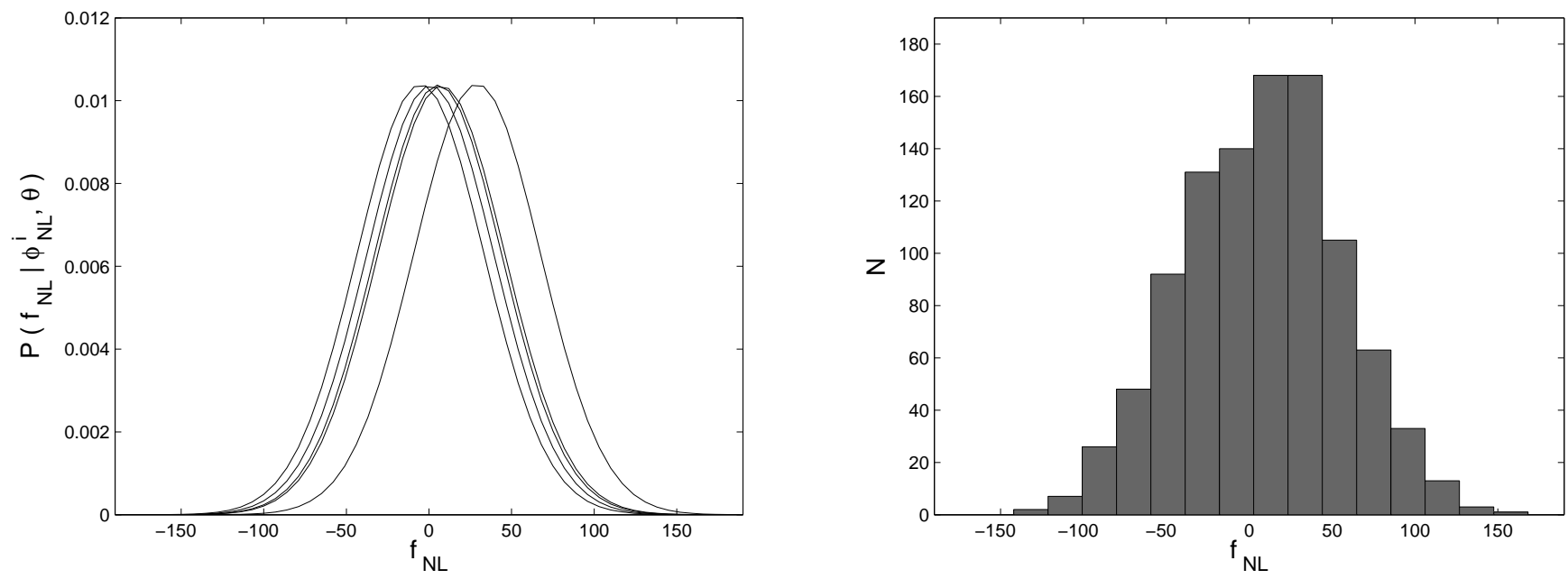

Fig. 3. Build-up of the posterior distribution of $f_{\mathrm{NL}}$. We depict the conditional probability distributions $P\left(f_{\mathrm{NL}} \mid \Phi_{\mathrm{NL}}, \theta\right)$ for several realizations of $\Phi_{\mathrm{NL}}$ (left panel) and the constructed posterior after 1000 drawn samples (right panel). The input parameters were chosen to be $N_{p i x}=10^{6}, f_{\mathrm{NL}}=0$, and $S / N=2$.

a mass and a momentum is assigned and the system is evolved deterministically from a starting point according to the Hamilton equations of motion.

The applicability of HMC sampling techniques to cosmological parameter estimation has been demonstrated in Hajian (2007), and the authors of Taylor et al. (2008) compared HMC with Gibbs sampling for CMB power spectrum analysis. To apply HMC sampling to $f_{\mathrm{NL}}$ inference, we deduced the expression of the Hamiltonian

$$
H=\sum_{i} \frac{p_{i}^{2}}{2 m_{i}}-\log \left[P\left(d, \Phi_{\mathrm{L}}, f_{\mathrm{NL}}, \theta\right)\right]
$$

where the potential is related to the PDF as defined in Eq. 8 . The Hamilton equations of motion,

$$
\begin{aligned}
\frac{\mathrm{d} x_{i}}{\mathrm{dt}} & =\frac{\partial H}{\partial p_{i}}, \\
\frac{\mathrm{d} p_{i}}{\mathrm{dt}} & =-\frac{\partial H}{\partial x_{i}}=\frac{\partial \log \left[P\left(d, \Phi_{\mathrm{L}}, f_{\mathrm{NL}}, \theta\right)\right]}{\partial x_{i}},
\end{aligned}
$$



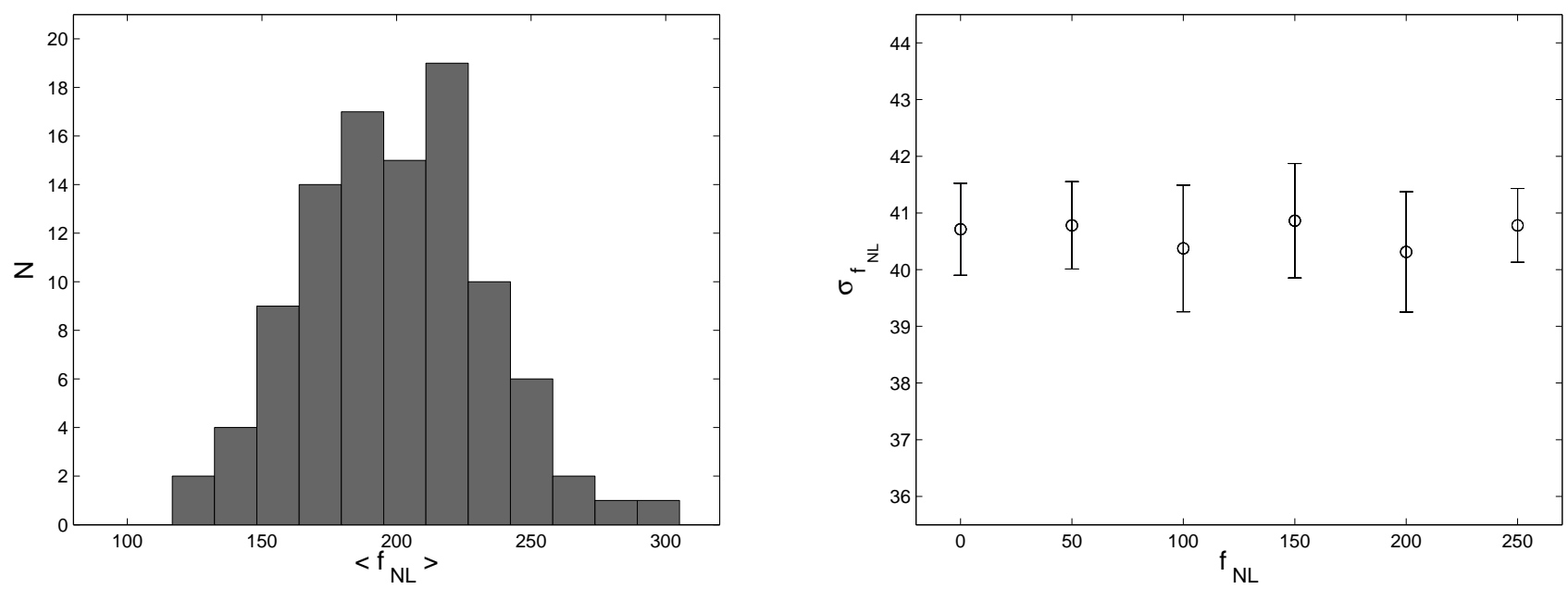

Fig. 4. Properties of the sampler. Left panel: Shown is the distribution of the derived mean values of $f_{\mathrm{NL}}$ from 100 simulations for a fiducial value of $f_{\mathrm{NL}}=200$. Right panel: We display the estimated standard deviation $\sigma_{f_{\mathrm{NL}}}$ of the drawn $f_{\mathrm{NL}}$ samples as a function of $f_{\mathrm{NL}}$. Each data point is averaged over 10 simulations. The input parameters used in this runs were $N_{p i x}=10^{6}$ and $S / N=10$, in each simulation 1000 samples were drawn.
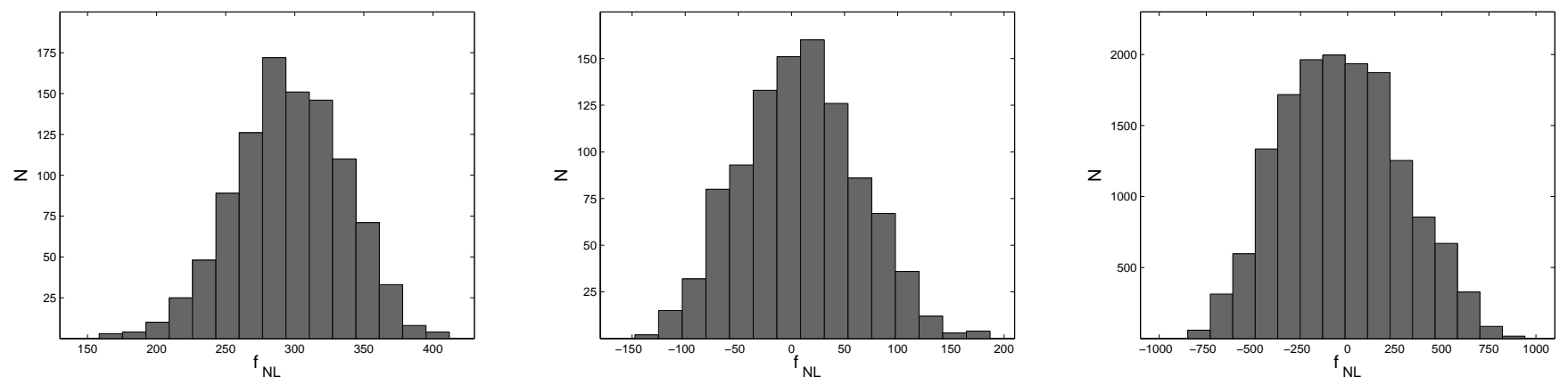

Fig. 5. Impact of the signal-to-noise ratio on the approximate sampling scheme. Left panel: Example of a constructed posterior distribution for $S / N=10$. Middle panel: Analysis of the same data set, but for $S / N=0.5$. At high noise level, the distribution becomes too narrow and systematically shifted towards $f_{\mathrm{NL}}=0$. Right panel: For comparison, we show the analysis of the data set at $S / N=0.5$ using exact Hamiltonian Monte Carlo sampling. As input parameters, we used $f_{\mathrm{NL}}=300$ and $N_{\text {pix }}=10^{6}$. For the approximate and exact analysis, 1000 and 15000 samples were drawn, respectively.
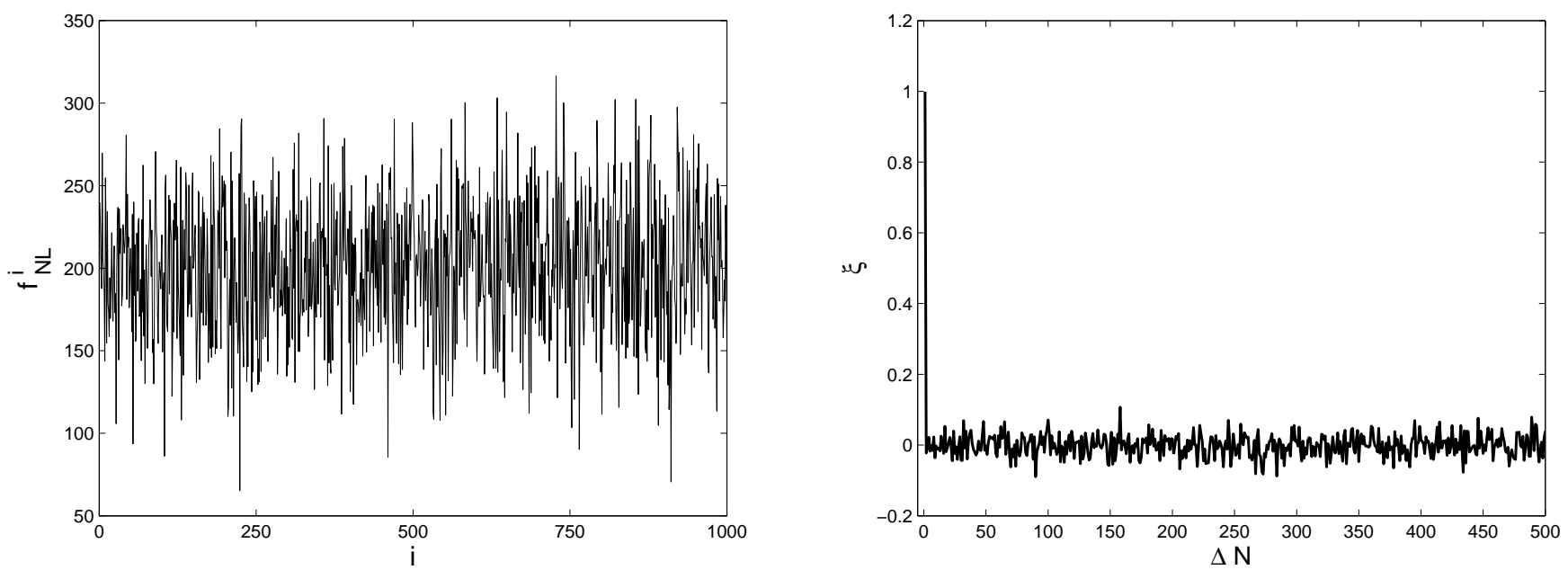

Fig. 6. Example $f_{\mathrm{NL}}$ chain. Left panel: We display the chain of 1000 generated $f_{\mathrm{NL}}$ samples which built up the histogram plotted on the right hand side in Fig. 2, Right panel: The autocorrelation function of $f_{\mathrm{NL}}$ confirms the uncorrelatedness of the samples. 

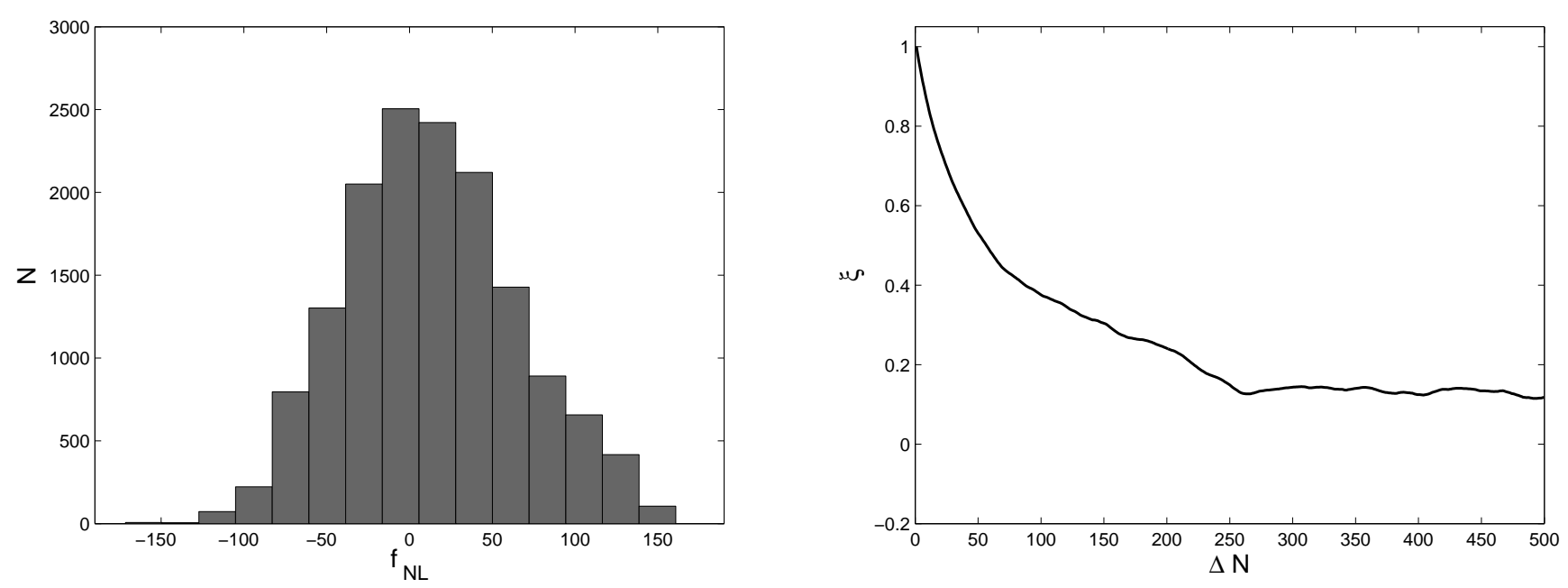

Fig. 7. Performance of the Hamilton Monte Carlo sampler. Left panel: Analysis of the data set of Fig. 3 using the HMC sampler. Here, 15000 samples were draw. Right panel: The autocorrelation function of $f_{\mathrm{NL}}$.

are integrated for each parameter $\left\{x_{i} ; p_{i}\right\}=\left\{f_{\mathrm{NL}}, \Phi_{\mathrm{L}} ; p_{f_{\mathrm{NL}}}, p_{\Phi_{\mathrm{L}}}\right\}$ using the leapfrog method with step size $\delta t$,

$$
\begin{aligned}
& p_{i}\left(t+\frac{\delta t}{2}\right)=p_{i}(t)+\left.\frac{\delta t}{2} \frac{\partial \log \left[P\left(d, \Phi_{\mathrm{L}}, f_{\mathrm{NL}}, \theta\right)\right]}{\partial x_{i}}\right|_{x(t)} \\
& x_{i}(t+\delta t)=x_{i}(t)+\frac{\delta t}{m_{i}} p_{i}\left(t+\frac{\delta t}{2}\right) \\
& p_{i}(t+\delta t)=p_{i}\left(t+\frac{\delta t}{2}\right)+\left.\frac{\delta t}{2} \frac{\partial \log \left[P\left(d, \Phi_{\mathrm{L}}, f_{\mathrm{NL}}, \theta\right)\right]}{\partial x_{i}}\right|_{x(t+\delta t)} .
\end{aligned}
$$

The equations of motion for $x_{i}$ are straightforward to compute, as they only depend on the momentum variable. To integrate the evolution equations for the $p_{i}$, we derive

$$
\begin{aligned}
& \frac{\partial \log \left[P\left(d, \Phi_{\mathrm{L}}, f_{\mathrm{NL}}, \theta\right)\right]}{\partial f_{\mathrm{NL}}}=\left(\Phi_{\mathrm{L}}^{2}-\left\langle\Phi_{\mathrm{L}}^{2}\right\rangle\right)^{\dagger} M^{\dagger} B^{\dagger} N^{-1}\left(d-B M \Phi_{\mathrm{L}}-f_{\mathrm{NL}} B M\left(\Phi_{\mathrm{L}}^{2}-\left\langle\Phi_{\mathrm{L}}^{2}\right\rangle\right)\right), \\
& \frac{\partial \log \left[P\left(d, \Phi_{\mathrm{L}}, f_{\mathrm{NL}}, \theta\right)\right]}{\partial \Phi_{\mathrm{L}}} \approx M^{\dagger} B^{\dagger} N^{-1}\left(d-B M \Phi_{\mathrm{L}}\right)-P_{\Phi}^{-1} \Phi_{\mathrm{L}}+2 f_{\mathrm{NL}} \operatorname{diag}\left(M^{\dagger} B^{\dagger} N^{-1} d\right) \Phi_{\mathrm{L}}+\mathcal{O}\left(\Phi_{\mathrm{L}}^{2}\right),
\end{aligned}
$$

where we have truncated the gradient in the latter equation at order $\mathcal{O}\left(\Phi_{\mathrm{L}}^{2}\right)$. The final point of the trajectory is accepted with probability $p=\min (1, \exp [-\Delta H])$, where $\Delta H$ is the difference in energy between the end- and starting point. This accept/reject step allows us to restore exactness as it eliminates the error introduced by approximating the gradient in Eq. 23 and by the numerical integration scheme. In general, only accurate integrations where $\Delta H$ is close to zero result in high acceptance rates. Furthermore, the efficiency of a HMC sampler is sensitive to the choice of the free parameters $m_{i}$, which corresponds to a mass. This issue is of particular importance if the quantities of interest possess variances varying by orders of magnitude. Following Tavlor et al. (2008), we chose the masses inversely proportional to the diagonal elements of the covariance matrix which we reconstructed out of the solution of the sampling scheme from Sect. 3. We initialized the algorithm by performing one draw of $\Phi_{\mathrm{NL}}$ from the conditional PDF $P\left(\Phi_{\mathrm{NL}} \mid d, \theta\right)$ and setting $f_{\mathrm{NL}}=0$. The outcome of repeated analyses of the data set presented in Fig. 3 is shown in Fig. 7 The consistency of the distributions confirms the equivalence of the two sampling techniques in the high signal-to-noise regime. However, convergence for the HMC is far slower, even for the idealized choice for $m_{i}$ and a reasonable starting guess, as can be seen from the large width of the autocorrelation function (see right panel of Fig. 17).

We conclude, therefore, that the direct sampling scheme presented in Sect. 3 is more efficient than HMC when applied to the detection of local non-Gaussianities in the high signal-to-noise regime. However, as shown in the rightmost panel of Fig. 15, the exact analysis using a HMC algorithm remains applicable at high noise level.

\section{Extension to realistic data}

Applying the method to a realistic CMB data set requires recovering the primordial potential $\Phi_{\mathrm{L}}$ on shells at numerous distances $r_{i}$ from the origin to the present time cosmic horizon. Thus, the product of the transfer matrix $M$ with the potential transforms to

$$
M \Phi \rightarrow \sum_{i} M_{i} \Phi\left(r_{i}\right) .
$$


Here, the matrix $M$ projects a weighted combination of the $\Phi\left(r_{i}\right)$ at different radii to a resulting two dimensional signal map $s$. The resolution of the $r$-grid can be coarser where the transfer functions for radiation are close to zero and must be finer at the distances of recombination and reionization. Another modification concerns the covariance matrix $P_{\Phi}$ of the potential. Now it additionally describes the correlation of $\Phi$ on distinct shells at different distances,

$$
\Phi^{\dagger} P_{\Phi}^{-1} \Phi \rightarrow \sum_{i, j} \Phi\left(r_{i}\right) P_{\Phi\left(r_{i}\right), \Phi\left(r_{j}\right)}^{-1} \Phi\left(r_{j}\right),
$$

and can be calculated from the primordial power spectrum $\mathcal{P}(k)$ predicted by inflation

$$
P_{\Phi\left(r_{i}\right), \Phi\left(r_{j}\right) \ell}=\frac{2}{\pi} \int k^{2} d k \mathcal{P}(k) j_{\ell}\left(k r_{1}\right) j_{\ell}\left(k r_{2}\right)
$$

To tighten the constraints on $\Phi$, polarization information can be included into the analysis as well simply by replacing the temperature by the polarization transfer function in the expression for $M$. We plan to study the application of our methods to realistic CMB data in a future publication.

The computational speed of a complete implementation is limited by harmonic transforms that scale as $\mathcal{O}\left(N_{p i x}^{3 / 2}\right)$.

\section{Summary}

In this paper, we developed two methods to infer the amplitude of the non-Gaussianity parameter $f_{\mathrm{NL}}$ from a data set within a Bayesian approach. We focused on the so called local type of non-Gaussianity and derived an expression for the joint probability distribution of $f_{\mathrm{NL}}$ and the primordial curvature perturbations, $\Phi$. Despite the methods are of general validity, we tailored our discussion to the case example of CMB data analysis.

We developed an exact Markov Chain sampler that generates correlated samples from the joint density using the Hamiltonian Monte Carlo approach. We implemented the HMC sampler and applied it to a toy model consisting of simulated measurements of a 1-D sky. These simulations demonstrate that the recovered posterior distribution is consistent with the level of simulated non-Gaussianity.

With two approximations that exploit the fact that the non-Gaussian contribution to the signal is next order in perturbation theory, we find a far more computationally efficient Monte Carlo sampling algorithm that produces independent samples from the $f_{\mathrm{NL}}$ posterior. The regime of applicability for this approximation is for data with high signal-to-noise and weak non-Gaussianity.

By comparison to the exact HMC sampler, we show that our approximate algorithm reproduces the posterior location and shape in its regime of applicability. If non-zero $f_{\mathrm{NL}}$ is not supported by the data the method is biased towards Gaussianity. The approximate posterior more strongly prefers zero $f_{\mathrm{NL}}$ compared to non-zero values than the exact posterior, as expected given the nature of the approximations which Gaussianize the prior. This method is therefore only applicable if the data contains sufficient support for the presence of non-Gaussianity essentially overruling the preference for Gaussianity in our approximate prior.

Our efficient method enables us to perform a Monte Carlo study of the behavior of the posterior density for our toy model data with high signal-to-noise per pixel. We found that the width of the posterior distribution does not change as a function of the level of non-Gaussianity in the data, contrary to the frequentist estimator where there is an additional, $f_{\mathrm{NL}}$ dependent, variance component (Creminelli et al. 2007; Liguori et al. 2007). Our results suggest that this may be an advantage of the Bayesian approach compared to the frequentist approach, motivating further study of the application of Bayesian statistics to the search for primordial local non-Gaussianity in current and future CMB data.

We close on a somewhat philosophical remark. Even though we chose a Gaussian prior approximation for expediency, it may actually be an accurate model of prior belief for many cosmologists since canonical theoretical models predict Gaussian perturbations. From that perspective our fast, approximate method may offer some (philosophically interesting) insight into the question "what level of signal-to-noise in the data is required to convince someone of the presence of non-Gaussianity whose prior belief is that the primordial perturbations are Gaussian?"

Acknowledgements. We thank the anonymous referee for the comments which helped to improve the presentation of our results. We are grateful to Rob Tandy for doing initial tests on detecting primordial non-Gaussianity in reconstructed sky maps. We thank Anthony J. Banday for useful conversations. BDW is partially supported by NSF grants AST 0507676 and AST 07-08849. BDW gratefully acknowledges the Alexander v. Humboldt Foundation's Friedrich Wilhelm Bessel Award which funded part of this work.

\section{References}

Acquaviva, V., Bartolo, N., Matarrese, S., \& Riotto, A. 2003, Nucl. Phys., B667, 119

Alishahiha, M., Silverstein, E., \& Tong, D. 2004, Phys. Rev. D, 70, 123505

Babich, D. 2005, Phys. Rev. D, 72, 043003

Babich, D., Creminelli, P., \& Zaldarriaga, M. 2004, Journal of Cosmology and Astro-Particle Physics, 8, 9

Bartolo, N., Komatsu, E., Matarrese, S., \& Riotto, A. 2004, Phys. Rep., 402, 103

Bean, R., Dunkley, J., \& Pierpaoli, E. 2006, Phys. Rev. D, 74, 063503

Bond, J. R., Jaffe, A. H., \& Knox, L. 1998, Phys. Rev. D, 57, 2117

Carvalho, P., Rocha, G., \& Hobson, M. P. 2009, MNRAS, 393, 681

Creminelli, P., Nicolis, A., Senatore, L., Tegmark, M., \& Zaldarriaga, M. 2006, Journal of Cosmology and Astro-Particle Physics, 5, 4

Creminelli, P., Senatore, L., \& Zaldarriaga, M. 2007, Journal of Cosmology and Astro-Particle Physics, 3, 19 
Duane, S., Kennedy, A. D., Pendleton, B. J., \& Roweth, D. 1987, Physics Letters B, 195, 216

Elsner, F. \& Wandelt, B. D. 2009, ApJS, 184, 264

Enqvist, K. \& Sloth, M. S. 2002, Nucl. Phys., B626, 395

Eriksen, H. K., Dickinson, C., Jewell, J. B., et al. 2008, ApJ, 672, L87

Eriksen, H. K., Dickinson, C., Lawrence, C. R., et al. 2006, ApJ, 641, 665

Gelman, A. \& Rubin, D. B. 1992, Statistical Science, 7, 457

Guth, A. H. 1981, Phys. Rev. D, 23, 347

Hajian, A. 2007, Phys. Rev. D, 75, 083525

Hinshaw, G., Weiland, J. L., Hill, R. S., et al. 2009, ApJS, 180, 225

Hobson, M. P. \& McLachlan, C. 2003, MNRAS, 338, 765

Jewell, J., Levin, S., \& Anderson, C. H. 2004, ApJ, 609, 1

Khoury, J., Ovrut, B. A., Steinhardt, P. J., \& Turok, N. 2001, Phys. Rev. D, 64, 123522

Komatsu, E. \& Spergel, D. N. 2001, Phys. Rev. D, 63, 063002

Komatsu, E., Spergel, D. N., \& Wandelt, B. D. 2005, ApJ, 634, 14

Lewis, A. \& Bridle, S. 2002, Phys. Rev. D, 66, 103511

Liguori, M., Yadav, A., Hansen, F. K., et al. 2007, Phys. Rev. D, 76, 105016

Linde, A. D. 1982, Physics Letters B, 108, 389

Lyth, D. H., Ungarelli, C., \& Wands, D. 2003, Phys. Rev. D, 67, 023503

Maldacena, J. M. 2003, JHEP, 05, 013

Moroi, T. \& Takahashi, T. 2001, Phys. Lett., B522, 215

Senatore, L. 2005, Phys. Rev. D, 71, 043512

Smith, K. M., Senatore, L., \& Zaldarriaga, M. 2009, Journal of Cosmology and Astro-Particle Physics, 9 , 6

Spergel, D. N., Bean, R., Doré, O., et al. 2007, ApJS, 170, 377

Steinhardt, P. J. \& Turok, N. 2002, Phys. Rev. D, 65, 126003

Taylor, J. F., Ashdown, M. A. J., \& Hobson, M. P. 2008, MNRAS, 389, 1284

Tegmark, M. 1997, Phys. Rev. D, 55, 5895

Trotta, R. 2007, MNRAS, 375, L26

Verde, L., Wang, L., Heavens, A. F., \& Kamionkowski, M. 2000, MNRAS, 313, 141

Wandelt, B. D., Larson, D. L., \& Lakshminarayanan, A. 2004, Phys. Rev. D, 70, 083511

Yadav, A. P. \& Wandelt, B. D. 2005, Phys. Rev. D, 71, 123004

Yadav, A. P. S., Komatsu, E., \& Wandelt, B. D. 2007, ApJ, 664, 680 